Reviews

\title{
Atherosclerosis and Matrix Dystrophy
}

\author{
Yoshiyuki Seyama and Hiroshi Wachi
}

Department of Clinical Chemistry, Hoshi University School of Pharmacy and Pharmaceutical Sciences, Tokyo, Japan.

\begin{abstract}
Atherosclerosis is characterized by inflammatory metabolic change with lipid accumulation in the artery. Atherosclerotic plaque occurs at discrete locations in the arterial system and involves the proliferation of smooth muscle cells (SMCs) together with imbalance of the extracellular matrix elements, elastic fiber in particular. The role of elastin in arterial development and disease was confirmed by generating mice that lack elastin. Thus, elastin is a critical regulatory molecule that regulates the phenotypic modulation, proliferation and migration of SMCs. We estimated that elastin expression and SMC proliferation are coupled inversely: potent stimulators of cell proliferation may potentially inhibit elastin expression and potent inhibitors of cell proliferation can stimulate elastin expression. Moreover, elastin was found to be expressed maximally at the $G_{0}$ and minimally at the $G_{2} / M$ phase during the cell cycle, suggesting that its expression is regulated by the cell growth state. The elastin peptide VPGVG enhanced SMC proliferation, resulting in the reduction of elastin expression. The inhibition of elastin expression by elastin fragments may be reflected in the negative feedback regulatory mechanism. The relationship between cell proliferation and elastin expression may be changed in atherosclerosis. Areas of atherosclerotic plaque show abnormality of elasticity and permeability from the viewpoint of the physiological function of the arterial wall. The etiology was estimated to be that cholesterol and calcium are deposited on the elastic fiber, resulting in decreased elastin synthesis and crosslinking formation. In addition, these dysfunctions of elastin fiber are also associated, in that the down-regulation of elastin and its related components (fibrillin-1 and lysyl oxidase) are directly related to calcification in SMCs. The denatured arterial elastin by cholesterol and calcium accumulation was also susceptible to proteolytic enzymes such as elastase and matrix metalloproteinase (MMP). Therefore, metabolic change in elastic fiber induces decreased elasticity and is associated with essential hypertension. Vitamin $\mathrm{K}_{2}$ is used in drug therapy against atherosclerosis, or calcification in diabetes mellitus or dialysis, due to its promotion of the carboxylation of the matrix Gla protein. J Atheroscler Thromb, 2004; 11: 236-245.
\end{abstract}

Key words: Atherosclerosis, Elastin, Extracellular matrix, Smooth muscle cell

\section{Vascular Connective Tissue (Vascular Extracellular Matrix) and Elastic Fiber}

The heart and arteries are always active mechanically and seem to wear out more than any other system or

Address for correspondence: Yoshiyuki Seyama, Department of Clinical Chemistry, Hoshi University School of Pharmacy and Pharmaceutical Sciences, 2-4-41 Ebara, Shinagawa-ku, Tokyo 1428501, Japan.

E-mail: seyama@hoshi.ac.jp

Received March 19, 2004.

Accepted for publication April 6, 2004. organs. The potential energy accumulated in the stretching of the vessel wall during contraction of the heart (systole) is dissipated in the elastic recoil of the wall during the period when the heart is inactive (diastole). This release of tension in the wall serves as an auxiliary pump, forcing the blood forward during diastole. Thus, near the heart, the flow of blood is intermittent and it is associated with the elasticity of the wall. The walls of the large conducting vessels make it possible to have a continuous flow with an intermittent pump. The normal human artery wall comprises endothelial cells with a few under- 
lying SMCs, which together make up the tunica intima. The underlying tunica media contains SMCs and their associated matrices only, and is separated from the intima by the internal elastic lamina, and from the surrounding adventitia, which contains fibroblasts, small feeding blood vessels, and adventitial nerves, by the external elastic lamina. In all blood vessels, the components of the connective tissue possess important structure and biomechanical functions. The lamellae of elastin are important in providing the elastomeric properties of larger arteries, and in the human aorta, elastin represent $20-50 \%$ of the total dry weight. Collagen is also present in a similar proportion in the human aorta, and provides tensile strength throughout the vascular system. Several different types of collagen have been isolated from blood vessels. The proportion of proteoglycan in the aorta is relatively smaller, about $1 \%$ of the dry weight, but even at this concentration the extended structures of these molecules are sufficient to fill the aqueous spaces of the tissues. Thin basement membranes are closely associated with both endothelial and smooth muscle cells. For the endothelium, the basement membrane is the site of cell attachment and also provides an attachment site for the connective tissue of the subendothelium, and to the elastin of the internal elastic lamina. It may act as a barrier to the passage of large macromolecules, such as lipoproteins, to the subendothelium. The basement membrane is composed of cell-attachment protein such as fibronectin, laminin, and type IV collagen. The cell-attachment protein contains one domain involved in binding the cell surface, and another domain for extracellular molecules $(1,2)$. Recently, Li et al. defined the role of elastin in arterial development and disease by generating mice that lack elastin (3). These mice die of obstructive arterial disease, which results from subendothelial cell proliferation and reorganization of smooth muscle. These cellular changes are similar to those seen in atherosclerosis. However, lack of elastin is not associated with endothelial damage, thrombosis or inflammation, which occur in models of atherosclerosis (4). Thus, elastin has an unanticipated regulatory function during arterial development, controlling the proliferation of smooth muscle and stabilizing the arterial structure. Moreover, Karnik et al. concluded that elastin is a critical regulatory molecule that controls the phenotypic modulation, proliferation and migration of vascular SMCs. This conclusion is based on the following results using vascular SMCs from mice lacking elastin. First, the cells lacking elastin proliferated at a rate greater than that of the wildtype cells. Second, the cells lacking elastin failed to form a mature contractile phenotype, which is characteristic of wild-type cells. Third, the cells lacking elastin migrated more aggressively to an exogenous chemoattractant than wild-type cells. Fourth, all of these aberrant phenotypes induced by the absence of elastin could be rescued in vitro by the addition of recombinant tropoelastin. Fifth, elastin exerted its regulatory function on SMCs by activating a G-protein coupled signaling pathway. Finally, restoring elastin to the site of vascular injury in vivo reduced the neointimal accumulation of vascular SMCs (5). Therefore, the purpose of this article is to review the literature regarding matrix, especially elastin, dystrophy during atherosclerosis.

Elastin fibers consist of two distinct components: elastin and microfibrils, the components of which include fibrillin, a microfibril-associated glycoprotein. Elastin is secreted from cells as a soluble protein of approximately $70 \mathrm{kDa}$ that undergoes a complex assembly process requiring the coordinate expression of microfibrillar proteins. Major types of domains are found in tropoelastin: hydrophobic domains rich in Gly, Val, Pro and Ala; and hydrophilic domains typically rich in Lys and Ala, involved in cross-linking. The C-terminus of tropoelastin is a highly basic amino acid residue (RKRK) and contains the only two Cys residues. The human single elastin gene has been localized to chromosome 7q11.1-7q21.1, spanning $45 \mathrm{~kb}$. The human gene has 34 exons with an intron: exon ratio of $20: 1$, indicating that relatively small exons are interspersed within a large intron. Alu repeats are found in the human elastin gene at a frequency four times higher than that elsewhere in the genome, raising the possibility of recombination events in this gene in the general population, and the possibility of its contribution to diseases such as supravalvular aortic stenosis. Some variation exists in the presence of exon homologues amongst species. For example, bovine exons 34 and 35 are absent from the human gene, while the human gene has the introduction of the unusual hydrophilic-encoding exon $26 \mathrm{~A}$, not described in any species. Exon 36 is highly conserved amongst species and codes for the C-terminus, in addition to a large $3^{\prime}$-untranslated region, suggesting the possibility of regulatory elements in this region. The decrease in elastin synthesis that occurs with age has been shown to be the result in part, of the destabilization of mRNA. Growth factors TGF- $\beta 1$ (6), IGF (7), IL-1 $\beta$ (8), and vitamin $D(9)$ have been shown to affect tropoelastin synthesis at either the promoter level or post-transcriptionally by affecting the stability of tropoelastin mRNA. The isolation of tropoelastin cDNAs indicates that variation is present within species in the nucleotide sequence and size of both the isolated mRNA and cDNA. These valuable cDNAs have been shown to be the result of alternative splicing of tropoelastin mRNA. At least $11 \mathrm{hu}-$ man tropoelastin splice variants have been identified with six exons, shown to be subject to alternative splicing exons 22, 23, 24, 26A, 32, and 33 (10). This alternative splicing is regulated by age- or disease-related changes in an isoform ratio. Translation of mRNA takes place on the surface of the rough endoplasmic reticulum (RER), and polypeptide chains are released into the lumen of the 
RER with the release of the signal peptide. Tropoelastin is secreted to the plasma membrane via secretory vesicles, and it is possible to accumulate tropoelastin in the RER and Golgi apparatus without modification such as glycation. Tropoelastin is soluble in cold aqueous solution of less than $20^{\circ} \mathrm{C}$. However, on raising the temperature towards the physiological range, the solution forms tropoelastin aggregates by interactions between hydrophobic domains (GVGVP, GGVP and GVGAP) in a process termed coacervation. Coacervation of tropoelastin is considered to be an important step in fibrillogenesis prior to cross-linking. The molecular events that lead to elastic fiber formation are not well understood. Morphological studies have demonstrated microfibril formation followed by an accumulation of tropoelastin, which gradually obscures the microfibrils as the fiber matures. These observations have led to the hypothesis that the microfibrillar network serves as a scaffold upon which tropoelastin is deposited and crosslinked. In the past few years, direct biochemical and immunological study has revealed a more accurate interaction between cells and fibrillin-1 or tropoelastin. The initial stages of fibrillin assembly occur at regions of the cell surface at which microfibrils appear attached to the cell membrane.

Fibrillin-1 and fibrillin-2 are highly homologous gene products with the same overall arrangement of repetitive domains. Structural information of fibrillin provided by analyses of the specific types of domains, such as calcium-binding EGF-like domains, 8-Cys domains, hybrid domains, and Pro/Gly-rich regions, will provide the basis for understanding molecular interactions (ligand interactions, binding to growth factors, and assembly of microfibrils). Fibrillin-1 has been found in every connective tissue examined. However, fibrillin-1-containing microfibrils are particularly abundant in the major tissues affected in Marfan syndrome. Moreover, in the adult aorta, fibrillin-1 is present in the intima, media and adventitia, whereas only a very small amount of fibrillin- 2 can be found in the media, and none in the adventitia or intima (11).

Using adhesion assays, Sakamoto et al. showed that cells interact with fibrillin-1 and that the RGD-containing domain defines a major cell-binding epitope (12). The integrin $\alpha_{v} \beta 3$ is the adhesion receptor responsible for RGD binding. An association with membrane-associated dense plaques has been observed for microfibrils that serve in cells as underlying elastic fibers. The propensity of cytoskeletal elements to cluster where microfibrils contact the cell surface suggests that protein components of the microfibril interact with cell-surface receptors, which serve as a transmembrane link between microfibrils and components of the cytoskeleton. After secretion into the extracellular space, tropoelastin is rapidly made insoluble by cross-link formation without proteolytic pro- cessing. The initial reaction is an oxidative deamination of Lys residues in tropoelastin by the lysyl oxidase to produce allysine ( $\alpha$-amino adipic $\delta$-semialdehyde). All subsequent reaction is a spontaneous condensation of closely positioned Lys and allysine residues, producing cross-links such as allysine aldol, lysinonorleucine, and merodesmosine, and tetra-functional cross-links such as desmosine and isodesmosine. In normal conditions, elastin is regulated by biosynthesis, maturation and degradation by enzymes and chemical mediators, while metabolic disorders of elastin and microfibrils in the arterial wall induce arterial disease such as atherosclerosis and aneurysm.

\section{Elastogenesis During Atherosclerosis}

\section{Regulation of elastin synthesis}

The early stage of atherosclerosis is characterized by excessive accumulation of intracellular and extracellular lipid deposits. Total collagen and elastin subsequently increase in the affected intima-media. The raised lesion may eventually become covered by a collagen fibrous cap. In more progressive phases of atherosclerosis with lipid deposition and calcification, the synthesis of collagen and elastin decreases in plaque, compared with that in normal regions. Therefore, the synthesis and degradation of extracellular matrix depends on the phase of atherosclerosis. We examined the correlation between elastin expression and proliferation of SMCs in cell culture (13). Elastin expression in cultured SMCs was enhanced by potent inhibitors of SMC proliferation, including retinoic acid (14) and heparin (15), while elastin expression was reduced by potent stimulators of SMC proliferation like EGF (16), angiotensin II (17) and high K concentration (18). These results suggest that elastin expression and SMC proliferation are coupled tightly and inversely: potent stimulators of cell proliferation may potentially inhibit elastin expression and potent inhibitors of cell proliferation can stimulate elastin expression. The number of local environment, extracellular matrix and intracellular interactions can also regulate the response to growth factor or cytokine. For example, nitric oxide (NO), an endothelium-dependent relaxing factor, continuously produces NO synthetase in the endothelium. Endothelial dysfunction causes decreased NO release, resulting in a shared process involving the pathogenesis of atherosclerosis and neointima formation after angioplasty. We demonstrated that NO inhibited SMC proliferation, while it enhanced expression of elastin (19). On the other hand, endothelin-1, a potent vasoconstrictor, has been isolated from cultured porcine aortic endothelial cells. Endothelin1 is known as a potent stimulator of cell proliferation and development of arterial diseases such as atherosclerosis. We demonstrated that endothelin-1 stimulated cell proliferation and suppressed the elastin expression, like 
EGF and angiotensin II. The inhibition of elastin synthesis was completely prevented by an endothelin receptor $A$ antagonist. These results indicate that endothelin- 1 can modulate elastin mRNA expression via an endothelin receptor A in cultured SMCs, and that regulators of elastin expression may play an important role in elastogenesis and SMC proliferation during the development of atherosclerosis (20). To elucidate the relationship between elastin expression and cell proliferation, elastin was found to be expressed maximally at the $\mathrm{G}_{0}$ and minimally at the $\mathrm{G}_{2} / \mathrm{M}$ phase during the cell cycle, suggesting that its expression is regulated by the cell growth state. Thus, the elastin gene could be a cell cycle-related gene. Under normal conditions, the vast majority of SMCs in the medial layer of the artery, elastin-rich tissue, are considered to be in a contractile state under the constraints of the extracellular matrix. The proliferation of SMCs has been reported to be selectively inhibited on $\alpha$-elastin-coated dishes. This suggests that SMCs can express a maximum level of elastin in the medial layer and that elastic fibers accumulated in the layer, in turn, can hinder rapid proliferation of SMCs. Thus, the preferential expression of elastin at the $G_{0}$ phase appears to be regulated, in part, by elastin molecules present in the extracellular space (21). Elastin has a unique repeating sequence in the hydrophobic region: tetrapeptide VPGX $(X=G$ or $A)$, pentapeptide VPGVG, hexapeptide XPGVGV (X = A or V), and nonapeptide VPGXGVGAG ( $\mathrm{X}=\mathrm{L}$ or F). Pentapeptide VPGVG is the only repeating sequence present in the elastin molecules of all animal species analyzed, including human, bovine, porcine and chicken. This sequence is active as a chemoattractant for monocytes, fibroblasts and tumor cells, and modulates protein kinase $\mathrm{C}$ activity in lung carcinoma cells. We examined in detail the relationship between elastin peptide and cell proliferation or elastin expression. The elastin peptide, VPGVG monomer and polymer, specifically enhanced SMC proliferation, resulting in the reduction of elastin expression. The inhibition of elastin expression by elastin fragments may reflect a negative feedback regulatory mechanism. This modulation may be important in maintaining a fine balance between synthesis and degradation of elastin in accumulative diseases such as atherosclerosis. Our results provide evidence that treatment of SMCs with the elastin fragment VPGVG resulted in the auto-regulation of elastin expression (22). Similar negative auto-regulation of elastin gene expression was indirectly suggested when elastin cross-linking formation was inhibited by treatment with $\beta$-APN (23) and when tropoelastin-derived degradation products were produced by pancreatic elastase (24). Moreover, similar autoregulation has also been reported in collagen synthesis, in which $\mathrm{N}$-propeptide of type I procollagen inhibited collagen synthesis and mRNA activities (25).

\section{Phenotypic change by extracellular matrix}

In a recent study, it was increasingly evident that arterial SMCs occur in two distinct states, a contractile and a synthetic phenotype (26). Contractile-state cells have a muscle-like appearance and contract in response to chemical and mechanical stimuli. Synthetic-state cells have a fibroblast-like appearance, and their function is to proliferate and produce extracellular matrix component. Contractile cells are able to change to the synthetic phenotype, and this appears to be an important early event in atherogenesis. Moreover, the synthetic phenotype is associated with increased binding and degradation of VLDL and increased binding of LDL. Conceivably, this reflects the increased need for cholesterol and other lipid components of membrane synthesis in the growing and rapidly proliferating synthetic-state cells. These results support the idea that the ability of SMCs to ingest and metabolize lipoproteins is correlated with their phenotypic state and replicative activity (27). As a detailed mechanism, Yamamoto et al. reported that extracellular matrix may control vascular responses in the differentiation of the phenotypic transition of SMC. Fibronectin and type I, III and V collagen promote the transition to the synthetic phenotype, while laminin, type IV collagen, elastin, heparin and heparin sulfate act in the opposite direction in phenotypic transition. The differential properties of SMCs are controlled by their adherence to these ECM components through specific cell surface receptors (integrin or non-integrin) (28).

\section{Accumulation of Lipid on Aortic Elastin}

Atherosclerosis is associated with local accumulation of lipid and calcium in atherosclerotic plaque. Areas of such plaque commonly show abnormality of elasticity and permeability from the viewpoint of the physiological function of the arterial wall. Since various plasma components may diffuse through the intima extracellular matrix, composed mainly of elastin, collagen, glycoprotein and glycosaminoglycan (GAG), there are several possible interactions between lipoproteins and extracellular matrix components that could result in altered lipoprotein metabolism in arterial wall cells. Nishida and Cogan (29) and Laverius (30) showed that positively $(+)$ charged groups on LDL from ions bound with negatively $(-)$ charged sulfate groups of GAGs. However, Kramsch et al. showed stable complexes between lipids and elastin by the hydrophobicity of the elastin protein, particularly in the presence of calcium ions (31). Noma et al. suggested that one of the mechanisms involved in the deposition of lipids in elastin may be an interaction of the elastin protein with serum LDL, resulting in a transfer of cholesterol ester to the elastin (32). Moreover, Noma et al. demonstrated a reduction of LDL binding by HDL that could have resulted from an impairment in the binding site in- 
volved in the direct chemical binding of lipids to the elastin protein molecule (33). In previous studies, the data indicated that local lipid deposition in atherosclerotic plaque is due to alteration of the elastin fraction, both quantitatively and qualitatively. We attempted to estimate the manner of lipid accumulation in the arterial connective tissues of human and experimental atherosclerosis. Therefore, arterial connective tissue-lipid complexes from the aorta were studied sequentially by treatment of nondelipidemic connective tissue with physical and chemical methods. The distribution of cholesterol in the aorta was mainly elastin fraction, compared to fractions of collagen, glycoprotein and glycosaminoglycan. Examination of the composition of each fraction, obtained by the present method, revealed that the glycosaminoglycan fraction contained mostly hexosamine, the glycoprotein fraction contained mainly sialic acid, the collagen fraction hydroxyproline and the elastin fraction only desmosine and isodesmosine. The cholesterol in the elastic fraction was digested by treatment with pancreatic elastase, which suggested that aortic cholesterol is mainly deposited on aortic elastic fiber and partially deposited on the aortic contamination material of elastic fiber. The lipid distribution in arterial elastin obtained from the experimental models (rabbit and rat) was similar to that in human atherosclerotic plaque; that is, an increased total cholesterol level in the elastin fraction is a common phenomenon in atherosclerosis (34).

To test the hypothesis that contact with elastin growth substrate increases the accumulation of cholesterol ester by SMCs and favors the formation of vacuolated lipidladen cells under conditions of hyperlipidemia, Grande et al. compared cholesterol ester synthesis by cells grown in plastic dishes with that by cells grown on elastin membranes, in the presence of normolipemic or hyperlipidemic serum, since pre-incubation of SMCs in lipoprotein-deficient serum has been utilized to up-regulate LDL receptors in order to increase cholesterol uptake and metabolism. Pre-incubation in lipoprotein-deficient serum enhanced the stimulatory effect of hyperlipidemic serum on cholesterol ester synthesis for the cells growing in plastic dishes but had a much less stimulatory effect on the cells growing on elastin membranes. This study indicated that a close association with elastin modulates the response of SMCs to hyperlipidemia and suggested a role for elastin in the formation of foam cells of smooth muscle origin in atherosclerosis (35).

The mode of binding of lipids to arterial elastin was tested by incubating defatted elastin protein with LDL. LDL with a high affinity for elastin was found deposited along with elastic fiber in atherosclerotic plaques. Thus, LDL may be involved in the inhibition of SMC migration induced by elastin. A previous study was designed to determine whether LDL acts on elastin-coated filters. Filters coated with elastin were treated with human plasma
LDL, and the effects of the pretreatment on the migration of cultured SMCs toward platelet-derived attractant in a Boyden chamber were examined. The migration was inhibited when the chamber was fitted with an elastincoated filter instead of a control filter. However, the cells migrated well when the Boyden chamber was fitted with an elastin-coated filter pretreated with LDL or anti-elastin antibody. The deposition of LDL in intima elastic fiber may foster medial SMC migration toward the intima (36). These findings suggest that the functional defense action of elastin against cholesterol synthesis and SMC migration is disturbed by accumulation of lipid on elastic fiber, and that the deposition on elastic fiber induces changes in the elasticity or physiological function of the arterial wall.

\section{Aortic Calcification}

Arterial calcification occurs with increasing age and in association with a diverse range of diseases, including atherosclerosis, diabetes, and uremia. It occurs at two sites, in the media, in which it is known as Monckeberg's sclerosis, and in the intima, in which it is invariably associated with atherosclerosis. Additionally, vascular calcification is an active process that has many similarities with ossification. Vascular calcification is now known to be present in $80 \%$ of significant lesions and in at least $90 \%$ of patients with coronary artery disease. Understanding the regulatory mechanisms of vascular calcification will allow future therapeutic approaches for preventing and possibly reversing this disease and its clinical consequences (37). In a population study, the value of pulse wave velocity (PWV), an indicator of vascular elasticity, increased with aging. PWV was positively associated with aging, calcification and the degree of atherosclerosis. On the other hand, PWV was negatively correlated with the contents of elastin (38). Thus, PWV has been used in clinical diagnosis in Japan, and these phenomena may suggest that calcification or calcium deposition inhibits elastin synthesis. Therefore, we examined qualitative changes in the elastin from calcified portions of human artery, compared to that at adjacent sites and in normal artery. The results from different sites of the calcified abdominal artery were as follows: There were more contents of $\mathrm{Ca}, \mathrm{P}$ and $\mathrm{Mg}$ in the aortic elastin fraction from the calcification site than in those at other sites. The content of desmosine, indicated as elastin content and a cross-link of elastin, in the calcification site was significantly lower than that in the other sites. In addition, its content was negatively associated with that of $\mathrm{Ca}$ and $\mathrm{P}$ in the elastin fraction. These results suggested that $\mathrm{Ca}$ and $\mathrm{P}$ deposition in elastin due to calcification cause as decrease in elastin or cross-links (desmosine) of elastin (39). Then, we examined the relationship between calcification in the aorta and elastin metabolism 
such as biosynthesis, maturation and degradation. An in vivo experiment, the aortic lysyl oxidase activity was significantly decreased in rats receiving atherogenic diet and Vit. $D_{2}$. The desmosine contents in the aortic elastin obtained from experimental arteriosclerosis induced by Vit. $D_{2}$ and atherogenic diet were lower than those in the control group. These results indicate that the decreased contents of cross-links (desmosine) in the experimental atherosclerosis are related to down-regulation of lysyl oxidase and elastin maturation during arteriosclerosis with calcification (40).

Recently, an in vitro aortic calcification system was developed as a useful model for analyzing the cellular mechanisms of calcification (41). We investigated the expression of major elastic fiber components such as tropoelastin, fibrillin-1, and an elastin-related enzyme, lysyl oxidase, in a calcification model using $\beta$-glycerophosphate ( $\beta$-GP) in cultured bovine aortic SMCs. $\beta-G P$ stimulated calcium deposition in a time-dependent manner. $\beta$-GP time-dependently decreased tropoelastin and fibrillin-1, as shown by Western blot analysis. The m-RNA levels of tropoelastin, fibrillin-1 and lysyl oxidase, assessed by reverse transcription-polymerase chain reaction, were also decreased by exposure to $\beta$-GP. Furthermore, we investigated whether the processes of calcification in SMCs directly control these regulations. In experiments using levamisole, an alkaline phosphatase inhibitor, and clodronate, a bisphosphonate, both inhibitors inhibited down-regulation during $\beta$-GP-induced calcification, suggesting that the down-regulation of tropoelastin, fibrillin-1 and lysyl oxidase is directly related to calcium deposition. In cases of vascular calcification, the decreased expression of tropoelastin, fibrillin-1 and lysyl oxidase may be partially responsible for decreased vascular elasticity and the decreased formation or maturation of elastin fiber (42).

An in vitro model of elastin fiber assembly in retinal pigmented epithelial cells (RPEs) has been developed as a useful model for analyzing the molecular and cellular mechanisms of elastic fiber assembly. RPEs express microfibrillar proteins (fibrillin-1 and microfibril-associated glycoprotein), but do not express tropoelastin. We examined the effect of tropoelastin on vascular calcification in cultured SMCs and RPEs. To understand the physiological function of tropoelastin in vascular calcification, we developed a calcification model of cultured RPEs. Inorganic phosphate induced calcium deposition, as assessed by von Kossa staining and $\mathrm{Ca}$ or $\mathrm{P}$ determination, in the RPEs, similar to that in SMCs. Moreover, osteopontin mRNA expression was up-regulated in the Pi-induced calcification of RPEs, while tropoelastin mRNA expression was decreased by calcium deposition in RPEs. Pi-induced calcification was partially suppressed after the addition of tropoelastin, due to elastic fiber formation. Therefore, tropoelastin and elastic fibers may contribute to the inhibition of vascular calcification via the 67-kDa elastin binding protein on the cell surface (43, 44). In addition to similar phenomena in endothelin-1, we demonstrated the expression of tropoelastin and lysyl oxidase at the gene level, as induced by endothelin-1, in cultured SMCs. Endothelin-1 stimulated cell proliferation and suppressed tropoelastin and lysyl oxidase mRNA expression (20). In atherosclerosis, abnormal cholesterol and calcium deposition in aortic elastin fiber is associated with alterations in the amino acid composition of elastin and cross-links. Moreover, these denatured elastin fibers may be susceptible to proteolytic enzymes, such as elastase and matrix metalloproteinase (MMP). In order to clarify the degradation of elastin under abnormal conditions, we examined the aortic elastolytic activity in rat experimental aneurysm induced by treatment with an inhibitor of lysyl oxidase ( $\beta$-aminopropionitrile: BAPN). There was a negative correlation between the elastolytic activity and the cross-link (desmosine) content in the aorta. The ratio of elastolytic activity to desmosine content in the aorta from treatment with BAPN increased compared with that of the control. We estimated that the decreased cross-linked elastin fiber under aneurysm and atherosclerosis was more susceptible by elastolytic enzymes (45). Deposition of lipid and minerals (calcium and phosphate) on the elastic fiber accompanied the splitting and fragmenting of the fiber.

According to a recent review, many kinds of extracellular matrixes are mainly produced by SMCs in both the normal arterial wall and atherosclerotic lesions. In particular, type I, III, IV, V, and VIII collagen, and elastin are expressed at a high level in atherosclerotic lesions. MMPs mainly produced by SMCs and macrophages are MMP-1, -2, -3, -7, -9 and -12 in the arterial wall. MMP$1,-2$, and -3 are highly expressed in the media of fetuses and neonates, but during aging, MMP-1 and -3 expression decreased $(46,47)$. Elevation of the blood level of MMP-2 and -9 is detected in acute coronary syndrome due to plaque rupture, but no direct causal relationship between MMP activity and plaque rupture has been demonstrated. These results suggest that denatured aortic elastin with lipid or calcification deposition is susceptible to elastolytic enzymes, elastase or MMPs $(48,49)$.

\section{Physiological Function (Vasodilatation) of Elastin}

Elastin is the core protein of elastic fibers and plays a role in determining the elasticity of the arterial wall. In cell-culture study, elastin is also considered to have biological effects on the migration, cell growth, and phenotypic changes of SMCs. Moreover, in vivo experimental studies have estimated the physiological function of elastin in arterial walls. Physiological degradation of elastin fibers, enhanced in vascular pathologies, leads to the presence of elastin peptides in the circulation, whih is 
increased in arteriosclerosis and aneurysm. Elastin peptides influence cell migration and proliferation, and induce endothelium-dependent vasodilatation mediated by NO. Elastin peptides act via binding to the 67-kDa subunit of the high affinity elastin -laminin receptor. The binding of elastin peptide to the $67-\mathrm{kDa}$ subunit of elastinlaminin receptor induces the activation of calcium membrane channels, resulting in an increase in both cytoplasmic- and nuclear-free calcium concentrations. The increased calcium leads to activation of NO synthetase and NO production in endothelial cells. Then, NO production induces a short-term vasorelaxation, counterbalancing the hypertensive and vasoconstrictive effect of atherosclerosis. Elastin peptides, circulating degradation products of extracellular matrix, could play a role in altering gene expression or influencing cell motility and differentiation (50).

Acquired elastic fiber abnormalities that alter the structure and mechanical properties of vessels are associated with aging and observed in vascular diseases such as atherosclerosis, aneurysm and hypertension. Faury et al. studied elastin insufficiency disease caused by lossof-function mutation or gene deletion in mice, and found that elastin haploinsufficiency results in cardiovascular hemodynamics and arterial wall structure. The animals were stable hypertensive with only mild cardiac hypertrophy, and did not exhibit the hypertension-induced arterial wall hypertrophy and decreased dispensability of large elastic arteries associated with essential hypertension. The association of hypertension with elastin haploinsufficiency in humans and mice strongly suggests that elastin and other proteins of elastic fiber should be considered causal for essential hypertension (51). Medley et al. examined whether genotypic variation in the extracellular matrix protein fibrillin-1 was associated with aortic stiffening and therefore could contribute to the cardiovascular risk associated with pulse pressure elevation in coronary disease. Although a causative link has not been shown, these data are consistent with the important role of the fibrillin-1 genotype in cardiovascular risk associated with large artery stiffening and pulse pressure elevation, in individuals with coronary disease (52). Moreover, Powell et al. reported that a strong interaction occurs between the fibrillin-1 genotype and blood pressure, contributing to the development of abdominal aortic aneurysm (53).

\section{Effect of Drugs on Arteriosclerosis}

\section{Elastase}

It has been strongly suggested that the deposition of lipids on the arterial wall in atherosclerosis is closely related to the metabolism of connective tissue. It is considered that an enzyme, elastase, regulates the metabolism of connective tissues. Lowering of elastase activity has been suggested to be an etiological factor of atherosclerosis. For this reason, elastase preparations (elaszymes) are externally administered to experimental animals or patients with atherosclerosis for basic and clinical studies. In this regard, Katayama reported that orally administered elastase was partially absorbed into the bloodstream (54). Externally administered elastase increased serum elastolytic activity and tended to decrease an accumulation of cholesterol in the rabbit aorta induced by cholesterol administration. Moreover, Hayashi et al. reported that elastase and/or feeding of a regression diet decreased wall stiffness and elastic modulus in the rabbit aorta, as assessed by pressure-diameter tests (55). These results suggest that externally administered elastase may improve the metabolism of denatured elastic fiber.

\section{Vitamin K}

It is well known that calcification of aortic tissue is one of the atherosclerotic processes of the blood vessel wall, and that the process becomes severe with advancing age. A population-based study indicated that women with aortic atherosclerosis have an impaired vitamin $\mathrm{K}$ status, as reflected by a lower nutritional vitamin $\mathrm{K}$ intake (56). It is known that one of the functions of vitamin $\mathrm{K}_{2}$ is to serve as a cofactor of the carboxylation of glutamate, making carboxyglutamate (Gla) by carboxylase. The Gla-containing proteins have a specific affinity for $\mathrm{Ca}$, and play regulatory roles in blood coagulation. Moreover, vitamin $\mathrm{K}_{2}$ seems to be essential for $\gamma$-carboxylation, which forms osteocalcin in bone and matrix Gla protein in soft tissue (the arteries and cartilage). Moreover, recent studies have demonstrated that the lack of matrix Gla protein caused calcification of the arteries and cartilage in mice. These results indicate that matrix Gla protein is an inhibitor or regulator of the calcification of soft tissue (57). An excess of vitamin $D_{2}$ or $D_{3}$ is known to cause Monckeberg's type arteriosclerosis with medial calcification, and to induce the calcification of soft tissues such as the heart and kidney (58). Vitamin $\mathrm{K}_{2}$ inhibited the increase in the aortic $\mathrm{Ca}$ and $\mathrm{P}$ induced by vita$\min D_{2}$, while there was no change due to Vitamin $K_{3}$ (containing a naphthoquinone ring) or geranylgeraniol (containing an aliphatic side chain) under experimental conditions. These results suggest that the anti-calcification or anti-atherosclerotic activity of vitamin $\mathrm{K}_{2}$ depends on a functional naphthoquinone ring and an aliphatic side chain (59). It is suggested that a mechanism of anti-calcification by vitamin $\mathrm{K}_{2}$ promoted the carboxylation of matrix Gla protein. Vitamin $\mathrm{K}_{2}$ also prevented the ectopic calcification accompanying with dialysis, diabetic mellitus and uremia. 


\section{References}

(1) Mayne R: Vascular connective tissue, Normal biology and derangement in human diseases. In: Connective Tissue Disease. Molecular Pathology of the Extracellular Matrix. ed by Uitto $\mathrm{J}$ and Perejda AJ, pp 163-183, Marcell Dekker, New York, 1987

(2) Hedin U: Extracellular matrix components and integrins in the control of arterial smooth muscle cell structure and function. J Atheroscler Thromb 1 (Suppl 1): S39-S46, 1994

(3) Li DY, Brooke B, Davis EC, Mecham RP, Sorensen LK, Boak BB, Eichwald E, and Keating MT: Elastin is an essential determinant of arterial morphogenesis. Nature, 393: 276-280, 1998

(4) Fan J and Watanabe T: Inflammatory reactions in the pathogenesis of atherosclerosis. J Atheroscler Thromb, 10: 63-71, 2003

( 5 ) Karnik SK, Brooke BS, Bayes-Genis A, Sorensen L, Wythe JD, Schwartz RS, Keating MT, and Li DY: A critical role for elastin signaling in vascular morphogenesis and disease. Development, 130: 411423, 2003

(6) Kucich U, Rosenbloom JC, Abrams WR, Bashir MM, and Rosenbloom J: Stabilization of elastin mRNA by TGF-beta: initial characterization of signaling pathway. Am J Respir Cell Mol Biol, 17: 10-16, 1997

( 7 ) Wolfe BL, Rich CB, Goud HD, Terpstra AJ, Bashir M, Rosenbloom J, Sonenshein GE, and Foster JA: Insulin-like growth factor-I regulates transcription of the elastin gene. J Biol Chem, 268: 12418-12426, 1993

( 8 ) Mauviel A, Chen YQ, Kahari VM, Ledo I, Wu M, Rudnicka L, and Uitto J: Human recombinant interleukin-1 beta up-regulates elastin gene expression in dermal fibroblasts. Evidence for transcriptional regulation in vitro and in vivo. J Biol Chem, 268: 6520-6524, 1993

(9) Pierce RA, Kolodziej ME, and Parks WC: 1,25Dihydroxyvitamin $D_{3}$ represses tropoelastin expression by a posttranscriptional mechanism. J Biol Chem, 267: 11593-11599, 1992

(10) Indik Z, Yeh H, Ornstein-Goldstein N, Kucich U, Abrams W, Rosenbloom JC, and Rosenbloom J: Structure of the elastin gene and alternative splicing of elastin mRNA: implications for human disease. Am J Med Genet, 34: 81-90, 1989

(11) Dazamaba B, Charbonneau N, Keene D, and Sakai L: Two fibrillins, multiple fibrillinopathies, how many functions? In: Extracellular Matrix-cell Interaction; Molecules to diseases. ed by Ninomiya $Y$, Olsen BR, and Ooyama T, pp 23-40, Japan Scientific Societies Press, Tokyo/S. Karger AG, Basel, 1998

(12) Sakamoto H, Broekelmann T, Cheresh DA, Ramirez F, Rosenbloom J, and Mecham RP: Cell-type spe- cific recognition of RGD- and non-RGD-containing cell binding domains in fibrillin-1. J Biol Chem, 271: 4916-4922, 1996

(13) Tajima S: Correlation of elastin expression and vascular smooth muscle cell proliferation in vitro. In: Extracellular Matrix-cell Interaction; Molecules to diseases. ed by Ninomiya Y, Olsen BR, and Ooyama T, pp 109-121, Japan Scientific Societies Press, Tokyo/S. Karger AG, Basel, 1998

(14) Hayashi A, Suzuki T, and Tajima S: Modulations of elastin expression and cell proliferation by retinoids in cultured vascular smooth muscle cells. J Biochem (Tokyo), 117: 132-136, 1995

(15) Wachi H, Seyama Y, and Tajima S: Modulation of elastin expression by heparin is dependent on the growth condition of vascular smooth muscle cells: up-regulation of elastin expression by heparin in the proliferating cells is mediated by the inhibition of protein kinase $\mathrm{C}$ activity. J Biochem (Tokyo), 118: 582-586, 1995

(16) Tokimitsu I, Tajima S, and Nishikawa T: Preferential inhibition of elastin synthesis by epidermal growth factor in chick aortic smooth muscle cells. Biochem Biophys Res Commun, 168: 850-856, 1990

(17) Tokimitsu I, Kato H, Wachi H, and Tajima S: Elastin synthesis is inhibited by angiotensin II but not by platelet-derived growth factor in arterial smooth muscle cells. Biochim Biophys Acta, 1207: 68-73, 1994

(18) Tokimitsu I and Tajima S: Inhibition of elastin synthesis by high potassium salt is mediated by $\mathrm{Ca}^{2+}$ influx in cultured smooth muscle cells in vitro: reciprocal effects of $\mathrm{K}^{+}$on elastin and collagen synthesis. J Biochem (Tokyo), 115: 536-539, 1994

(19) Sugitani H, Wachi H, Tajima S, and Seyama Y: Nitric oxide stimulates elastin expression in chick aortic smooth muscle cells. Biol Pharm Bull, 24: 461464, 2001

(20) Wachi H, Sugitani H, Tajima S, and Seyama Y: Endothelin-1 down-regulation expression of tropoelastin and lysyl oxidase mRNA in cultured chick aortic smooth muscle cells. J Health Science, 47: 525-532, 2001

(21) Wachi H, Seyama Y, Yamashita S, and Tajima S: Cell cycle-dependent regulation of elastin gene in cultured chick vascular smooth-muscle cells. Biochem J, 309 (Pt 2): 575-579, 1995

(22) Wachi H, Seyama Y, Yamashita S, Suganami H, Uemura $\mathrm{Y}$, Okamoto K, Yamada $\mathrm{H}$, and Tajima S: Stimulation of cell proliferation and autoregulation of elastin expression by elastin peptide VPGVG in cultured chick vascular smooth muscle cells. FEBS Lett, 368: 215-219, 1995

(23) Jackson LE, Faris B, Martin BM, Jones HV, Rich 
CB, Foster JA, and Franzblau C: The effect of betaaminopropionitrile on elastin gene expression in smooth muscle cell cultures. Biochem Biophys Res Commun, 179: 939-944, 1991

(24) Tajima S, Wachi H, and Seyama Y: Tropoelastinderived degradation products downregulate eastin expression in vascular smooth muscle cell in culture. Connect Tissue, 28: 231-219, 1996

(25) Horlein D, McPherson J, Goh SH, and Bornstein P: Regulation of protein synthesis: translational control by procollagen-derived fragments. Proc Natl Acad Sci USA, 78: 6163-6167, 1981

(26) Glukhova MA, Frid MG, and Koteliansky VE: Phenotypic changes of human aortic smooth muscle cells during development and in adult. J Atheroscler Thromb, 1 (Suppl 1): S47-S49, 1994

(27) Thyberg J, Hedin U, Sjolund M, Palmberg L, and Bottger BA: Regulation of differentiated properties and proliferation of arterial smooth muscle cells. Arteriosclerosis, 10: 966-990, 1990

(28) Yamamoto K, Yamamoto M, Yamamoto N, and Aoyagi M: Regulation of differentiated properties of vascular smooth muscle cells in atherosclerosis: Role of extracellular matrix. Connect Tissue, 34: 317-325, 2002

(29) Nishida T and Cogan U: Nature of the interaction of dextran sulfate with low density lipoproteins of plasma. J Biol Chem, 245: 4689-4697, 1970

(30) Iverius $\mathrm{PH}$ : The interaction between human plasma lipoproteins and connective tissue glycosaminoglycans. J Biol Chem, 247: 2607-2613, 1972

(31) Kramsch DM, Franzblau C, and Hollander W: The protein and lipid composition of arterial elastin and its relationship to lipid accumulation in the atherosclerotic plaque. J Clin Invest, 50: 1666-1677, 1971

(32) Noma A, Takahashi T, Yamada K, and Wada T: Elastin-lipid interaction action in the arterial wall. Part 1. Extraction of elastin from human aortic intima. Atherosclerosis, 33: 29-39, 1979

(33) Noma A, Takahashi T, and Wada T: Elastin-lipid interaction in the arterial wall. Part 2. In vitro binding of lipoprotein-lipids to arterial elastin and the inhibitory effect of high density lipoproteins on the process. Atherosclerosis, 38: 373-382, 1981

(34) Seyama Y, Hayashi M, Usami E, Tsuchida H, Tokudome S, and Yamashita S: Basic study on nondelipidemic fractionation of aortic connective tissue of human and experimental atherosclerosis. Jpn J Clin Chem, 19: 53-61, 1990

(35) Grande J, Davis HR, Bates S, Mathews MB, and Glagov S: Effect of an elastin growth substrate on cholesteryl ester synthesis and foam cell formation by cultured aortic smooth muscle cells. Atherosclerosis, 68: 87-93, 1987

(36) Ooyama T, Sakamoto H, Fukuda K, Oda H, Murai
Y, Seyama Y, and Nakamura H: LDL eliminates the inhibitory effect of human aortic elastin on cultured rat aortic smooth muscle cell migration in vitro. J Jpn Atheroscler Soc, 20: 703-707, 1992

(37) Parhami F, Bostrom K, Watson K, and Demer LL: Role of molecular regulation in vascular calcification. J Atheroscler Thromb, 3: 90-94, 1996

(38) Hasegawa M, Kawasaki T, Arai C, Kashiwakura Y, Kishi Y, and Yoshimura S: Clinical application of aortic pulse wave velocity (PWV) method to evaluate the effect of antisclerotic agent (Elaszym). J Jpn Atheroscler Soc, 8: 419-423, 1980

(39) Hayashi M, Osakabe T, Ushio F, and Seyama Y: Qualitative change in the elastin from calcified portion of human artery. Jpn J Gerial, 36: 404-407, 1999

(40) Hashimoto S, Seyama Y, Yokokura T, and Mutai $\mathrm{M}$ : Effects of chlorella phospholipid on the aortic collagen and elastin metabolism and on the serum lipid content in rats with experimental arteriosclerosis. Exp Mol Pathol, 37: 150-155, 1982

(41) Shioi A, Nishizawa Y, Jono S, Koyama H, Hosoi M, and Morii $\mathrm{H}$ : Beta-glycerophosphate accelerates calcification in cultured bovine vascular smooth muscle cells. Arterioscler Thromb Vasc Biol, 15: 2003-2009, 1995

(42) Sugitani H, Wachi H, Mecham RP, and Seyama Y: Accelerated calcification represses the expression of elastic fiber components and lysyl oxidase in cultured bovine aortic smooth muscle cells. J Atheroscler Thromb, 9: 292-298, 2002

(43) Sugitani $H$, Wachi $H$, Murata $H$, Sato F, Mecham $\mathrm{RP}$, and Seyama Y: Characterization of an in vitro model of calcification in retinal pigmented epithelial cells. J Atheroscler Thromb, 10: 48-56, 2003

(44) Wachi H, Sugitani H, Murata H, Nakazawa J, Mecham RP, and Seyama Y: Tropoelastin inhhibits vascular calcification via $67 \mathrm{kDa}$ elastin binding protein in cultured bovine aortic smooth muscle cells. J Atheroscler Thromb, 11: 159-166, 2004

(45) Kobayashi T, Osakabe T, and Seyama Y: Comparison of elastolytic activity between experimental aneurysm and experimental diabetes mellitus. Biol Pharm Bull, 21: 775-777, 1998

(46) Kuzuya M and Iguchi A: Role of matrix metalloproteinases in vascular remodeling. J Atheroscler Thromb, 10: 275-282, 2003

(47) Okamoto Y, Satomura K, Ohsuzu F, Nakamura H, Takeuchi $\mathrm{K}$, and Yoshioka M: Expression of matrix metalloproteinase-3 in experimental atherosclerotic plaques. J Atheroscler Thromb, 8: 50-54, 2001

(48) Katsuda S and Kaji T: Atherosclerosis and extracellular matrix. J Atheroscler Thromb, 10: 267-274, 2003

(49) Yano T, Kawano H, Mochizuki H, Doi O, Nakamura $\mathrm{T}$, and Saito $\mathrm{Y}$ : Atherosclerotic plaques composed 
of a large core of foam cells covered with thin fibrous caps in twice-injured carotid arterial specimens obtained from high cholesterol diet-fed rabbits. J Atheroscler Thromb, 7: 83-90, 2000

(50) Faury G, Usson Y, Robert-Nicoud M, Robert L, and Verdetti J: Nuclear and cytoplasmic free calcium level changes induced by elastin peptides in human endothelial cells. Proc Natl Acad Sci U S A, 95: 2967-2972, 1998

(51) Faury G, Pezet M, Knutsen RH, Boyle WA, Heximer SP, McLean SE, Minkes RK, Blumer KJ, Kovacs A, Kelly DP, Li DY, Starcher B, and Mecham RP: Developmental adaptation of the mouse cardiovascular system to elastin haploinsufficiency. J Clin Invest, 112: 1419-1428, 2003

(52) Medley TL, Cole TJ, Gatzka CD, Wang WY, Dart AM, and Kingwell BA: Fibrillin-1 genotype is associated with aortic stiffness and disease severity in patients with coronary artery disease. Circulation, 105: 810-815, 2002

(53) Seyama $Y$ and Yamashita S: Effect of elastase on the metabolism of lipids and connective tissues of vascular wall in experimental atherosclerosis. In: Vascular Neuroeffector Mechanisms 4th International Symposium. ed by Bevan JA, Fujikawa M, Maxwell RA, Mori K, Shibata S, and Toda N, pp 377-388, Raven Press New York, 1983
(54) Katayama K and Fujita T: Studies on biotransformation of elastase. III. Effects of elastase binding proteins in serum on the diappearance of $131 \mathrm{I}$-labeled elastase from blood. Biochim Biophys Acta, 336: 165-177, 1974

(55) Hayashi K, Takamizawa K, Nakamura T, Kato T, and Tsushima N: Effects of elastase on the stiffness and elastic properties of arterial walls in cholesterol-fed rabbits. Atherosclerosis, 66: 259-267, 1987

(56) Luo G, Ducy P, McKee MD, Pinero GJ, Loyer E, Behringer RR, and Karsenty G: Spontaneous calcification of arteries and cartilage in mice lacking matrix GLA protein. Nature, 386: 78-81, 1997

(57) Seyama $Y$, Horiuch $M$, Hayashi $M$, and Kanke $Y$ : Effect of vitamin $\mathrm{K}_{2}$ on experimental calcinosis induced by vitamin $D_{2}$ in rat soft tissue. Int $\mathrm{J}$ Vitam Nutr Res, 66: 36-38, 1996

(58) Seyama $Y$, Hayashi M, Takegami $H$, and Usami E: Comparative effects of vitamin $\mathrm{K}_{2}$ and vitamin $\mathrm{E}$ on experimental arteriosclerosis. Int J Vitam Nutr Res, 69: 23-26, 1999

(59) Seyama Y, Kimoto S, Marukawa Y, Horiuchi M, Hayashi M, and Usami E: Comparative effects of vitamin $\mathrm{K}_{2}$ and estradiol on experimental arteriosclerosis with diabetes mellitus. Int J Vitam Nutr Res, 70: 301-304, 2000 